\title{
Prevención de drogas.
} Buenas prácticas de trece programas de Colombia

\author{
Drug Prevention. An Overview \\ of thirteen Colombian Programs
}

\section{Prevenção de drogas. Boas práticas \\ de treze programas da Colômbia}

José Silverio Espinal Bedoya, Psic ${ }^{1 *}$;

Gustavo Adolfo Calderón Vallejo, Soc²

Recibido: 01 de agosto de 2017 / Aceptado: 10 de enero de 2018

Dor: http://dx.doi.org/10.12804/revistas.urosario.edu.co/revsalud/a.7269

Para citar este artículo: Espinal-Bedoya JS, Calderón-Vallejo GA. Prevención de drogas. Buenas prácticas de trece programas de

Colombia. Rev Cienc Salud. 2018;16(3):534-49. Dor: http://dx.doi.org/10.12804/revistas.urosario.edu.co/revsalud/a.7269

\section{Resumen}

Introducción: se describen las buenas prácticas de prevención, distinguidas de esta manera por 13 programas de prevención de drogas en Colombia. La identificación de buenas prácticas se ha extendido en el campo de las drogas para ayudar a mejorar los procesos, la toma de decisiones, encontrar metodologías exitosas y, en general, mejorar el saber hacer en los programas de drogas. Para la investigación se realizó un estudio cualitativo, hermenéutico, con alcance descriptivo, a partir de un proceso de realización de entrevistas a coordinadores y operadores de dichos programas, información que se sometió a un análisis de categorías previas y emergentes. Desarrollo: los resultados mostraron que algunas de las buenas prácticas descritas en la literatura especializada eran implementadas por los programas consultados, aunque no todas las necesarias para mostrar resultados eficaces y comprobables, especialmente en la generación de evidencia. Conclusiones: de forma aislada, dichas prácticas no tienen la suficiente fuerza; pero, si fueran adoptadas en su conjunto redundarían en una mejora de la prevención de drogas en Colombia.

Palabras clave: uso indebido de drogas, prevención primaria, promoción de la salud, consumidores de drogas, abuso de drogas, drogadicción.

\section{Abstract}

Introduction: There is a description of good practices in drug prevention, acknowledged as such by 13 drug prevention programs in Colombia. The identification of good practices has been widened in the field of drugs in order to improve processes, decision making, finding successful methodologies and in general improving the know-how processes in drug programs. A qualitative study was carried out under a descriptive hermeneutic approach based on the information gathered through interviews with 
program coordinators and staff; the information was analyzed and categorized. Development: The results showed that the programs that were consulted used some of the good practices that were described in the specialized literature, even though not all were necessary to show efficient results, especially in generating evidences. Conclusions: When isolated, such practices are not enough, if they are adopted as a whole they would improve drug prevention processes in Colombia.

Keywords: Drug misuse, primary prevention, health promotion, drug consumers, drug abuse, drug addiction.

\section{Resumo}

Introdução: descrevem-se as boas práticas de prevenção distinguidas desta maneira por 13 programas de prevenção de drogas na Colômbia. A identificação de boas práticas se tem estendido no campo das drogas para ajudar a melhorar os processos, a tomada de decisões, encontrar metodologias bem-sucedidas e em geral melhorar o saber fazer nos programas de drogas. Para a pesquisa se realizou um estudo qualitativo, hermenêutico, com alcance descritivo, a partir de um processo de realização de entrevistas a coordenadores e operadores de ditos programas, informação que se submeteu a uma análise de categorias prévias e emergentes. Desenvolvimento: os resultados mostraram que algumas das boas práticas descritas na literatura especializada eram implementadas pelos programas consultados, ainda que não todas as necessárias para mostrar resultados eficazes e comprováveis, especialmente na geração de evidência. Conclusões: de forma isolada ditas práticas não têm a suficiente força; mas se fossem adotadas em seu conjunto redundariam em uma melhora da prevenção de drogas na Colômbia.

Palavras-chave: uso indevido de drogas, prevenção primária, promoção da saúde, consumidores de drogas, abuso de drogas, drogadição.

\section{Introducción}

E $_{\text {consumo de sustancias psicoactivas (SPA), ya no como una estrategia mediática, sino como }}^{\text {s deconocimiento internacional la importancia de los programas de prevención del }}$ una serie de estrategias basadas en la evidencia científica con el objetivo de generar un desarrollo seguro de los niños y jóvenes (1). Colombia, en particular, adopta una postura congruente con los lineamientos internacionales y, mediante su Política Nacional de reducción de la demanda de drogas, asume esta tendencia en el marco de la protección social; esto es, que más allá de solo evitar o prevenir el uso indebido de sustancias psicoactivas, tiene en cuenta condiciones de calidad de vida y el bienestar en los niveles individual, micro y macrosocial (2).

Sin embargo, la prevención no siempre sucede de acuerdo con los lineamientos y estándares internacionales. Por ello, esta investigación se propuso poder identificar aquellas prácticas que 13 programas de prevención de diversas instituciones de Colombia llevan a cabo para la prevención del consumo de drogas en el país, poniendo especial atención en las buenas prácticas, con el fin de identificar aquellas que, según los participantes de esta investigación, han resultado útiles en la consecución de sus objetivos. Ello reviste un interés particular como aporte para el desarrollo de programas de prevención en el contexto colombiano. 
El concepto de buenas prácticas es muy utilizado en el campo de las drogas, tanto en tratamiento como en prevención, reducción de daños e inclusión social de personas consumidoras. Ya desde 1990, la Organización Mundial de la Salud (oms) comienza a incorporarlo en el campo del tratamiento (3). En general, este concepto ha sido utilizado para referirse a las formas de hacer que ayudan a mejorar los procesos, la toma de decisiones, el mejoramiento de la calidad de vida de las personas que muestran efectos positivos en la población objeto de un programa, que han mostrado sostenibilidad, innovación e impacto, entre otros atributos, los cuales han sido tenidos en cuenta en diversos catálogos y manuales internacionales, y publicaciones que han intentado recoger las mejores prácticas en el campo de las drogodependencias, uno de los más importantes lo tiene el Observatorio Europeo de Drogas (EMCDDA) (4). Así, también, sobre todo en España, hay diversos catálogos y guías que han intentado recoger las buenas prácticas de aspectos tan diversos como la inclusión laboral de personas que han pasado por procesos de tratamiento de drogas, prevención y redes sociales, drogodependencias en general, entre otros temas (5-7).

Otros atributos que los anteriores autores señalan, que se espera tengan las buenas prácticas en el campo de las drogas, es que estas sean sistematizadas; es decir, que impliquen una metodología que intenta descubrir cómo mejorar el saber hacer y que sean trasferibles, lo cual implica la posibilidad que para otros sea una práctica replicable. Por consiguiente, aunque pareciera un concepto moral (lo bueno), en realidad, definir qué es o no una buena práctica pasa por la aplicación de criterios técnicos y no por una valoración a priori.

La identificación y valoración de buenas prácticas tienen su valor en que son obtenidas por medio de la voz de los actores que vivieron la experiencia, siendo por tanto una comunicación de la experiencia entre pares, en la que se transmite un conocimiento de relevancia social que resulta útil a los profesionales que desarrollan prácticas similares (8). Esto cobra sentido en esta investigación, como ya se mencionó, en recoger la visión de los actores participantes como aportes para el contexto colombiano.

Los trece programas de prevención del consumo de sPA, objeto de este estudio, estaban ubicados en cuatro ciudades capitales de Colombia (Bogotá, Medellín, Cali y Bucaramanga), durante 2016. Aunque se encontraron programas del ámbito familiar, laboral y comunitario, el grueso de los programas tenía su actuación en el sector escolar, tanto de primaria, secundaria como en universitarios. Al observar la tendencia marcada de la población a que se dirigen son programas para público joven. Los objetivos de los programas eran muy diversos: evitar el consumo, retrasar la edad de inicio, evitar el abuso en el consumo de sustancias, evitar daños asociados y fortalecer habilidades para la vida.

En cuanto a los enfoques, sobresalieron los programas basados en la salud pública y específicamente los que parten de identificar factores de riesgo y protección, para desde allí evitar y/o disminuir el consumo de sustancias psicoactivas y retrasar la edad de inicio, pero al tiempo incidir en las problemáticas relacionadas como delincuencia, violencia y deserción 
escolar, entre otras. También había otros enfoques a nivel de manejo de autoesquemas, desarrollo de identidad y el fortalecimiento de habilidades cognitivas, de manejo de emociones y habilidades sociales. Por último, se menciona el modelo ecológico basado en los cambios del ser humano y modificaciones de su entorno.

Metodológicamente se desarrolló una investigación con un enfoque cualitativo y específicamente una modalidad de estudio hermenéutico, con un alcance principalmente descriptivo. Las categorías previas de las que partió el estudio fueron: programas de prevención, modelos de prevención, estrategias de prevención, necesidades preventivas y prácticas de prevención, siendo esta última categoría de la que versa este artículo.

La modalidad del estudio hermenéutico, se entendió como la construcción de un texto social, a partir de la palabra de los actores consultados, para ser interpretado por el grupo de investigación y realizar una triangulación con la literatura universal sobre el tema, esta información recolectada permitió nutrir la discusión del artículo.

Los participantes fueron los coordinadores de los programas de prevención contactados en cada institución y que accedieron voluntariamente a brindar la información sobre las características, los contenidos y métodos de sus programas. También se tuvieron en cuenta a quienes estaban encargados de implementar los programas en sus respectivos ámbitos de actuación; a ambos tipos de participantes se les aplicó consentimiento informado.

Como técnica de recolección de información se eligió la entrevista semiestructurada, por dar la posibilidad de un acercamiento lo más natural posible, pero con mucho detenimiento, a lo que son los programas de prevención, sus modelos y sus prácticas. Se construyeron dos instrumentos, uno para los coordinadores de los programas y el segundo para los operadores de estos; con los instrumentos se indagaba por las acciones que ellos consideraban buenas prácticas, los estándares de calidad y evidencia y tipo de estrategias implementadas.

Los hallazgos de la presente investigación muestran grandes diferencias entre los programas contemplados, entre otras cosas por dificultades en aspectos metodológicos, contextuales y de presupuesto. Por tanto, las conclusiones aquí señaladas no significan que todos los programas contengan todos los atributos de buenas prácticas que se identificaron, sino que algunos tienen unas y otros, otras. No obstante, dichos programas en su conjunto dejan ver algunos elementos de buenas prácticas, que, aunque pocos de ellos las desarrollan completamente, si fueran aplicados de manera sistemática y constante podrían representar un gran avance en materia de prevención para Colombia.

A partir del análisis categorial realizado, este artículo muestra hallazgos de buenas prácticas de prevención de drogas relacionadas con: (a) la relación usuario y aplicador del programa, (b) la integralidad de este, (c) las temáticas y técnicas desarrolladas y (d) los criterios de calidad y evidencia que conducían a buenas prácticas. 


\section{Desarrollo}

\section{La relación interpersonal entre ejecutores y beneficiarios del programa preventivo}

Se destaca cómo algunos informantes le dan mucha importancia a generar estrategias que permitan el acercamiento con la población, de forma que el programa de prevención sea aceptado por esta. En ese sentido, poder establecer un vínculo interpersonal se considera fundamental para su desarrollo, siendo esto una especie de prerrequisito para que funcione; por tanto, no son solo las características técnicas las que lo hacen funcionar, sino que la calidad de la relación entre quienes implementan el programa y quienes se benefician de él es fundamental, incluso haciendo cosas que no están contenidas en los protocolos de trabajo.

Así, entonces, entre las prácticas que ayudan a generar y mejorar la calidad del vínculo entre quien aplica el programa y quien lo recibe se encuentran: tener un personaje imaginario para acercarse a los niños; llamar a los participantes para ver cómo están, por qué no han vuelto, motivarlos para el siguiente encuentro o preguntarles por la tareas que se han dejado para la casa; apoyar las escuelas con acciones que están por fuera del programa; ayudar a los participantes en otras necesidades (orientación a un servicio, por ejemplo) e incluso regalos.

La relación entre aplicador y beneficiario, como una forma de favorecer condiciones para que el programa sea aceptado, es todavía un área por explorar y a la que los programas consultados le dan mucha importancia, pues sin esta condición difícilmente habrían podido ejecutarse, por lo cual no ha de valorarse como un asunto de menor importancia. Existen estudios y meta-análisis sobre la relación entre el tipo de aplicador (si es profesional de la salud o profesor) y los resultados. Estos muestran que hay evidencia contradictoria en cuanto a la eficacia según el tipo de aplicador, pero para el asunto en mención señalan que más que el tipo de aplicador se deben analizar sus competencias, por ejemplo, las relaciones con los estudiantes, lo que apoya la idea que emerge en los programas analizados sobre implementar prácticas que mejoren la relación aplicador y beneficiario $(9,10)$. Sin embargo, un estudio reciente sobre un programa en Colombia destaca cómo la formación a educadores en estrategias de prevención facilita su implementación (11).

\section{Sobre la integralidad de los programas de prevención de drogas}

Otro elemento que sale a relucir tiene que ver con la integralidad de las intervenciones, referidas a la necesidad de tener múltiples componentes para intervenir en diferentes variables al mismo tiempo, con el fin de lograr inclusión social, permitirle a la población acceso a diferentes rutas en asuntos de salud, educación, trabajo y satisfacción de sus derechos, 
promover el trabajo comunitario para dejar una capacidad instalada y empoderamiento para encontrar soluciones a sus necesidades. Se muestra, además, la necesidad de integración de la familia en los procesos de sus hijos.

Esta visión de integralidad es, sin embargo, cuestionada. Aunque hay estudios que dicen que los programas de múltiples componentes son eficaces para la prevención del alcohol y, según Jones, los programas que involucran comunidad, escuela y familia tienen resultados satisfactorios, para el EMCDDA hay pocas pruebas de que sean más efectivos que aquellos de un solo componente $(4,12,13)$. Los programas consultados en esta investigación resaltan la importancia de apuntar a diferentes factores de riesgo y protección como el acceso a la educación, la salud y el trabajo, la resolución de conflictos familiares, los derechos humanos, entre otros, como objetivos de la acción preventiva. Esto lo relacionan con las dificultades de la población con que trabajan, la cual además está afectada por diversas situaciones de vulnerabilidad, marginalidad y exclusión social y en distintos grados de riesgo, que hacen que el problema del consumo sea más complejo. Al respecto, un meta-análisis sobre la efectividad de los programas de prevención escolares de tabaco, alcohol y cannabis muestra que el impacto de los programas de prevención escolares es consistente, aunque limitado, pues existen muchos factores de tipo social que influyen en el consumo y, por tanto, limitan el impacto (14). Otro estudio sobre los programas de prevención escolares más efectivos ha evidenciado que estos se centran en factores de riesgo y protección psicosocial presentes en la primeras etapas de consumo (15). De otra parte, un estudio sobre un programa de prevención del consumo de sustancias psicoactivas para población infantil ha identificado la obtención de resultados importantes debido a haber tenido en cuenta elementos tales como influencias sociales, interacción y alternativas al consumo, lo que podría mostrar, por un lado, que los programas deben atender diversas variables, pues dichos factores guardan relaciones entre sí, y, de otro lado, que no pueden centrarse solo en la sustancia en sí misma, sino en los factores que propician su consumo (11). Así mismo, sistemas de prevención comunitaria que involucran diversos componentes se han mostrado como altamente promisorios para el contexto latinoamericano (16).

Además, algunas organizaciones describen los beneficios de intervenir sobre diversos factores de riesgo psicosocial y propiciar cambios en el ambiente, las políticas, las comunidades, etc. $(1,17,18)$. Por tanto, la prevención no puede agotarse en los métodos más tradicionales que abogan por las condiciones del sujeto y, especialmente, su cognición y por los asuntos meramente relacionados con el consumo de drogas. Esto conduce a la consideración de que el consumo de drogas no pasa solo por una decisión reflexiva, sino que está mediado por asuntos como el ambiente, las emociones y las normas sociales, y que por consiguiente su intervención no puede estar conducida solo por estrategias que acuden a la consciencia (la cognición) y centradas en el individuo (19). 
Todas estas conclusiones dan pie a pensar que no se pueden hacer solo intervenciones de tipo programático (una serie de sesiones de talleres) sino también de tipo sistémico, es decir, que intervengan en las diferentes variables asociadas con el consumo y no solo en las de tipo personal, como suelen hacerlo la mayoría de programas de prevención, lo que respaldaría la práctica de los programas consultados que trabajan sobre diferentes variables a la vez y que involucran intervenciones tales como refuerzo escolar, formación y gestión para el acceso al trabajo, mejoramiento de las relaciones familiares y orientación o acompañamiento para recibir atención médica, legal o humanitaria, entre otros. Puede decirse que estas intervenciones van orientadas a mejorar la red de apoyo de las personas intervenidas; esto es, poder generar interconexiones entre las personas y su comunidad que favorezcan los procesos de inclusión social y por consiguiente cierto estado de soporte que contribuya a modificar los procesos de salud/enfermedad $(20,21)$.

La oms recomienda que la prevención eficaz debe integrar el ámbito individual, comunitario y ambiental (17). La United Nations Office on Drugs and Crime (unoDc) señala la importancia de intervenciones basadas en la evidencia que apunten a múltiples ámbitos y vulnerabilidades, entendiendo la complejidad del problema que no puede ser afrontado mediante una sola estrategia preventiva (1). Sobre esto, se puede observar también una tensión entre los lineamientos que provienen de organismos internacionales, como los mencionados anteriormente, y lo que dice parte de la evidencia científica; es decir, aunque esta, como ya se mencionó, sostiene que los programas multicomponentes no tienen pruebas de ser más efectivos, los organismos internacionales y algunas experiencias específicas (tanto las consultadas en la literatura, como las de esta investigación) tienden a recomendar no centrar la prevención en un solo aspecto.

\section{Técnicas y temáticas desarrolladas}

Respecto a las técnicas y temáticas desarrolladas, las buenas prácticas son mencionadas por los informantes de esta investigación como aquellas que han generado buenos resultados, entre las que se encuentran: las actividades lúdicas y vivenciales, la mediación social, es decir, la formación de estudiantes y profesores para que desarrollen acciones de prevención en el contexto escolar y los talleres. También resaltan aquellas que realizan prácticas para que los maestros se motiven y acompañen el aprendizaje de sus estudiantes. Otros utilizan material educativo, la lúdica, las técnicas vivenciales, los talleres reflexivos. Algunos ven en la expresión de afectos una forma de enganche a los procesos. Dentro de las temáticas más comunes están las habilidades para la vida, pautas de crianza, hábitos de vida saludables y convivencia. Otros programas tienen en cuenta la atención de casos como una de sus estrategias para intervenir de forma selectiva e indicada a quienes presentan mayores factores de riesgo. Esta forma de prevención se apoya en gran medida en posibilitarles a los participantes 
una conexión con las redes de apoyo, a lo que algunos llaman “relación de ayuda”, mediada en algunos casos por los pares.

Puede decirse que, en general, estas técnicas buscan conducirse de manera interactiva, lo que según algunos meta-análisis, es considerado como una buena práctica, pues los métodos interactivos, que incluyen técnicas como el debate y el juego de roles, se muestran más eficaces que los no interactivos, ya que estimulan el intercambio de ideas y el aprendizaje; incluso existen estudios en los que el tipo de metodología tuvo más peso en el impacto que los contenidos $(15,22)$.

Sobre los temas abordados, en relación con las habilidades para la vida, se encuentran en la literatura especializada conclusiones que se contradicen entre ellas; algunos autores relatan que estos programas suelen mostrar buenos resultados (15, 23, 24). Sin embargo, cuestionan que programas como Life Skills Training sean capaces de reducir el consumo de marihuana en adolescentes (25). Respecto a las pautas de crianza, se ha mostrado que las acciones orientadas a mejorar la relación familiar, la comunicación y la supervisión parental son efectivas en los programas de prevención familiar (26). Así también, intervenir sobre aspectos relacionados con la convivencia son señalados como buenas prácticas, pero sin existir un buen soporte de evidencia sobre ello; sin embargo, los factores de riesgo de tipo comunitario, como, por ejemplo, la deprivación económica, desorganización comunitaria, leyes comunitarias favorables a la criminalidad, que se trabajan desde un enfoque de salud pública tienen alta relación con esta forma de proceder (27).

En general, las técnicas y temáticas utilizadas por los programas consultados están dirigidas a buscar cambios en los individuos para tratar de persuadir la decisión de consumir drogas o generar destrezas que les permitan en un momento determinado rechazar la oferta de consumirlas. No obstante, este enfoque es limitado, según lo ya señalado sobre las dificultades de basarse en métodos que acuden predominantemente a la cognición y la necesidad de acudir a estrategias más integrales, lo que de alguna manera resulta ser un contradicción en los programas analizados, pues aunque los informantes describen la complejidad de las diversas necesidades de la población afectada, predomina la práctica de centrar la prevención principalmente en el individuo y su parte cognitiva (19).

\section{Los criterios de calidad y evidencia}

Respecto a la generación de calidad y evidencia, es importante tener en cuenta que los resultados de las evaluaciones de programas de prevención, en general, tienen algunas limitaciones; por un lado, porque no abarcan todo tipo de intervenciones, habiendo muchos más resultados en los programas escolares. Además, la mayoría de estos estudios se han hecho en los Estados Unidos, lo que genera dudas sobre su aplicabilidad en otros contextos; incluso existen limitaciones para afirmar que un programa se convierta en modelo de prevención, 
pues la evidencia no es suficiente ni consistente (28). Esto implica que, para el caso de los programas de prevención en Colombia, aplican las mismas restricciones, sumándole que estos corresponden tanto al contexto escolar como al laboral y comunitario.

En esta categoría se indagó sobre las prácticas que implementan los programas de prevención de drogas para asegurar la mejor calidad posible y si siguen recomendaciones basadas en evidencia científica, siendo estos aspectos donde más deficiencias se encontraron, lo cual es señalado por los mismos participantes de la investigación. Los hallazgos tienen que ver con la fidelidad en la aplicación de los programas preventivos, su estructura, los equipos de trabajo que los implementan y las bases científicas y métodos para recoger evidencia.

En torno a la elección del equipo que desarrolla las actividades preventivas, las organizaciones valoran la diversidad de orientaciones teóricas de los aspirantes y el deseo de trabajar en prevención; una organización incluso valora mucho las condiciones personales en relación con el consumo de drogas. Se resalta la realización de formación del equipo de manera interna o con formadores externos, como práctica que ayuda a mejorar la calidad de los programas.

También consideran que los programas son estructurados cuando tienen un conjunto de acciones relacionadas entre sí, que no obedecen a criterios personales del desarrollador del programa y que no son acciones aisladas o circunstanciales. Destacan que haya unas fases claramente definidas; que pueda comprenderse el proceso por el cual se llegará a los objetivos deseados; que haya involucramiento de diferentes actores, conocimiento previo de los riesgos y la población y sensibilización a esta sobre el programa; que las intervenciones sean constantes y repetitivas; que haya registros del proceso.

Respecto a la fidelidad de la aplicación, varios programas manifiestan tener mecanismos para hacer que se desarrollen cumpliendo con los criterios previamente establecidos y no se desvíen de la intencionalidad prevista. Algunos lo hacen de manera previa, es decir, generando guías o protocolos que los facilitadores deben seguir y otros incluso verifican que lo hagan. Algunos programas son muy estrictos en que los criterios se cumplan lo más cercano posible a lo predefinido, y otros son más laxos y permiten que se hagan adaptaciones según criterios de los facilitadores del programa. Las prácticas que se desarrollan para evidenciar esto son: manuales de procedimientos, protocolos de trabajo, entrenamiento de facilitadores, supervisión de facilitadores, seguimiento de logística de las actividades.

Hay una tensión que sale en la investigación entre el ajuste a la fidelidad del programa o hacer adaptaciones contextuales; entre quienes abogan por el cumplimiento estricto de los criterios y metodologías y quienes lo hacen por una construcción del mismo de acuerdo a las características propias de la comunidad con que se aplica. En general, la alta fidelidad es defendida más en los programas importados, aunque cuestionada por quienes aplican 
el programa en campo, y la adaptación contextual es promovida por los programas de tipo comunitario. La fidelidad se ha descrito de gran importancia, pues cuando un programa se desarrolla sin seguir los estándares que la investigación sobre este ha generado puede ser inefectivo (29).

Aunque se encontraron pocas buenas prácticas para generar evidencia, los participantes aceptan la falencia y destacan la importancia de incorporarla a sus programas. Al respecto se encuentra que algunos incluyen criterios de evidencia que no son elaboración propia, sino producidos por otros programas o investigaciones, con la idea de generar los mismos impactos que estos han obtenido. Otros se basan en criterios propios del programa que solo pueden señalar por medio de la observación o de mecanismos como aplicación de pretest y postest, registros cualitativos y cuantitativos de las actividades desarrolladas, incluso pruebas de orina. También se acude a la narración de los participantes acerca de los impactos que dicen haber tenido. Las organizaciones realizan ejercicios de evaluación internos más orientados a los procesos, con el objetivo de mejorarlos. Algunos han podido generar información, principalmente, sobre resultados de variables intermediadoras. Solo en un programa, a partir de la información recolectada, probablemente podrán generar evidencia, pues está en curso una evaluación de impacto y hay otro que ha aplicado una línea de base, realizado encuestas para medición de resultados y tiene una batería de indicadores para evaluar el desarrollo del programa; con esto manifiesta tener evidencia de cambios. Se observa que hay quienes intentan generar información con medios técnicos para que en un futuro el programa pueda ser evaluado, otros lo hacen de forma intuitiva a partir de sus observaciones. También es llamativo un caso en el que se implementa la evaluación como mecanismo de retroalimentación, es decir, los instrumentos de observación que aplican permiten tomar decisiones sobre qué temas deben desarrollarse en cada grupo en particular y a la vez obtener información sobre los resultados.

Los que manifestaron no evaluar, aducen que no se ha contemplado por falta de tiempo (pues los aplicadores del programa, además, hacen otro tipo de actividades), por discontinuidad de las intervenciones y/o por falta de financiación.

En una revisión de algunos estudios longitudinales sobre programas de prevención de drogas, los cuales fueron realizados con seguimiento a cuatro años, se encuentra que la metodología más utilizada ha sido estudios experimentales con aplicación de línea de base para determinar el estado inicial de la población intervenida y luego una evaluación de cierre para comparar cambios. Estos estudios han mostrado algunos resultados importantes. Gómez, Luengo y Romero, en un estudio realizado en la Comunidad Autónoma Gallega con una muestra de 1029 adolescentes, evidenciaron que el grupo intervenido presentó un menor consumo de tabaco y alcohol en el seguimiento a un año; sin embargo, en las evaluaciones siguientes los efectos disminuyeron, aunque hay diferencias significativas en el consumo de otras sustancias como el cannabis (30). A una conclusión similar 
llegó otro estudio de Fraguela, Martin y Trinanes, en este caso, en la Ciudad de Santiago de Compostela (31). Por su parte, estudios longitudinales de intervenciones basadas en el programa LifeSkills mostraron resultados significativos en cuanto a una menor probabilidad de usar drogas ilícitas, frente a las personas que no recibieron el programa, incluso uno de estos estudios ha hecho seguimiento por 12 años $(23,32)$. También encontraron resultados en jóvenes con alto riesgo de consumo de sustancias psicoactivas y disminución en la probabilidad de realizar violaciones de tránsito $(33,34)$.

Otro estudio de tipo longitudinal, que buscaba cambiar comportamientos hacia hábitos de vida saludables en alumnos de 12 a 16 años de niveles educación secundaria, logró mostrar cambios de actitud favorecedores de la prevención (35). También se han logrado cambios positivos en programas realizados con niños y niñas en contextos de vulnerabilidad en cuanto a la autoestima (conocimiento de sí mismo), cuidados de la salud y habilidades para la vida (36). Estos hallazgos son consistentes con algunas de las estrategias implementadas en los 13 programas objeto de esta evaluación. Dichos efectos son, en general, pequeños, situación que también ha sido referenciada por otros autores, quienes afirman que dada esta situación, los programas de prevención de drogas en ámbitos escolares deberían formar parte de estrategias más integrales, apreciación que también concuerda con las posturas de algunos de los programas participantes en esta investigación, los cuales abogan por estrategias multicomponentes que intervengan en diversos factores de riesgo y ámbitos a la vez (37).

Para Comas, tener criterios de evidencia no depende de que así lo haya dicho la comunidad científica, sino de las evidencias generadas, y estas no obedecen solo al hecho de tener un experimento o ensayo aleatorio, sino que es algo más complejo que depende de unos estándares que tienen que ver con que haya un número suficiente de ensayos (principio empírico) donde se reiteren los procedimientos metodológicos, tener bases muestrales representativas, contrastar diferentes métodos para evitar sesgos o conclusiones previamente esperadas, garantizar la aleatoriedad y no confundirla con el azar, someter las hipótesis y los hallazgos a un modelo racional y teórico, es decir, no basta con lo que los datos aportan si estos no son contrastados, además, la evidencia debe poder ser comprobada en la práctica por quienes implementan los programas de prevención y, finalmente, debe aplicar criterios de falsabilidad, no presentándose como verdad absoluta e inamovible, que no permita la construcción futura de conocimiento (38). Teniendo en cuenta estos criterios, se encuentra que, a los programas consultados en esta investigación, se les hace difícil llevarlos a cabo por las múltiples limitaciones que tienen, y que es un enfoque limitado pensar la evaluación y generación de evidencia a partir de prácticas como la aplicación de pretest y postest sin un adecuado engranaje de estos estándares. 
Las prácticas de calidad y evidencia encontradas, tales como las estrategias para lograr la fidelidad en la aplicación de los programas o los métodos para para recoger evidencia, salvo excepciones, son fragmentarias y resultan insuficiente para dar cuenta del impacto, así como para cumplir con exigencias de estándares actuales de calidad y evidencia tales como el Programa de Cooperación entre América Latina, el Caribe y la Unión Europea en Políticas sobre Drogas copolad (2014), unodc (2015) o EMCDDA (2016) [1, 4, 28].

Para generar evidencia, aspectos como la rigurosidad en la evaluación para que los tomadores de decisiones conozcan los resultados, la comprensión científica de las vulnerabilidades, una evaluación que permita saber si los programas son adecuados a los nuevos contextos no están presentes en la mayoría de programas analizados en este estudio (1). Igual sucede con criterios tales como la identificación de un modelo teórico explicativo de los consumos de drogas, objetivos definidos con base en necesidades previamente identificadas y con indicadores basales, intensidad y duración adecuada, existencias de planes de evaluación de resultados, entre otros (28).

Sin embargo, es importante señalar que uno de los elementos que no contribuye a la generación de evidencia en los programas de prevención de drogas en Colombia tiene que ver con la sostenibilidad, pues, aunque existiera la disposición para evaluarlos, el hecho de que estos sean a corto plazo, interrumpidos y con insuficientes recursos, hace difícil la tarea de generar evidencia. Al respecto unodc dice que la prevención de drogas eficaz y rentable necesita de políticas con inversiones a mediano y largo plazo, lo que implica tener mecanismos de revisión y ajuste de sistemas nacionales de prevención, recursos suficientes, recopilación de datos constantes, apoyo a la investigación para la evaluación, cualificación de los profesionales que intervienen en la planificación, ejecución, seguimiento y evaluación de las estrategias de prevención de drogas (1).

Finalmente, es importante dejar en consideración cierta tensión existente entre las buenas prácticas y la recolección de evidencia científica. El EMcDDA hace énfasis en que las buenas prácticas estén sustentadas en la mejor evidencia disponible, la cual debe estar respaldada en investigaciones científicas $(4,5)$. Sin embargo, otros consideran que son una alternativa frente a las dificultades que en muchas ocasiones presenta la aplicación de las evaluaciones de resultados, la estandarización o modelización de estrategias y las dificultades metodológicas de aplicación de evaluaciones por método experimentales en algunas situaciones; además, que si se considera que las buenas prácticas se restrinjan exclusivamente a la mejor evidencia disponible, ello descartaría otros elementos necesarios para la mejora de los procesos y la toma de decisiones $(3,5)$. 


\section{Conclusiones}

ibien, los programas de prevención analizados tienen en cuenta algunas buenas prácticas

Sque están referenciadas en la literatura especializada en el tema, estas no son suficientes para que cada programa pueda mostrar resultados eficaces y comprobables. Sin embargo, si estas prácticas fueran aplicadas en conjunto podrían redundar en un mejor ejercicio de prevención en el contexto colombiano.

Aunque existe poca evidencia, se destaca la importancia de generar una buena relación interpersonal entre quién aplica el programa y sus beneficiarios, de lo cual depende en gran medida el logro de los resultados esperados, siendo este un asunto que requiere mayor profundización en la literatura universal.

La integralidad de las intervenciones parece ser un tema de vital importancia, dada la complejidad del fenómeno y de las poblaciones a las cuales acceden los programas de prevención analizados, los cuales, además del consumo de drogas, se enfrentan a diferentes situaciones que ponen en vulnerabilidad a las personas usuarias de estos programas.

La mayoría de programas de prevención acuden al desarrollo de temáticas y estrategias que principalmente buscan generar cambios de tipo cognitivo, lo que resulta ser un enfoque insuficiente dada la complejidad del fenómeno señalado.

Siendo la aplicación de criterios de calidad y evidencia lo que mostró dificultades en los programas consultados, se resalta la necesidad de adopción de mejores prácticas en esta materia que respalden los resultados que estos programas puedan estar generando.

Los programas de prevención en Colombia se enfrentan a una serie de dificultades que los limitan para obtener mejores resultados y esto tiene que ver en gran medida con su poca sostenibilidad, la cual se relaciona con recursos financieros insuficientes y aplicaciones cortas e interrumpidas.

\section{Descargos de responsabilidad}

— ste artículo es producto de una investigación más amplia denominada "Prevención del consumo de sustancias psicoactivas en Colombia. Programas, enfoques y prácticas", desarrollada entre las universidades Luis Amigó y San Buenaventura, la Corporación Surgir y la Alcaldía de Medellín. Las fuentes de financiación del proyecto fueron internas, colocadas por las instituciones a donde pertenecen los autores. La responsabilidad por lo dicho en el artículo es solo de los autores.

Se aplicó consentimiento informado, el cual cumple con los requisitos exigidos en el artículo 15 de la resolución N. 008423 de 1993, expedida por el Ministerio de Salud, y de 
acuerdo con el Artículo 11 de esta, el estudio se clasifica en la categoría de "Investigación con riesgo mínimo”, dado que no se manipula la conducta de los participantes.

\section{Referencias}

1. United Nations Office on Drugs and Crime (UNODC). International Standars on Drug Use Prevention [internet]. 2015 [citado 2017 may 18]. Disponible en: https://www.unodc.org/ documents/prevention/UNODC_2013_2015_international_standards_on_drug_use_prevention_E.pdf

2. Ministerio de la Protección Social, Dirección General de Salud Pública. Política nacional para la reducción del consumo de sustancias psicoactivas y su impacto [internet]. 2007 [citado 2017 ene 30]. Disponible en: https://www.odc.gov.co/Portals/1/Docs/politDrogas/ politica_nacional_consumo.pdf

3. Uchtenhagen A. ¿Qué significa ‘mejores prácticas’ en el tratamiento de las toxicomanías? Revista Española de drogodependencias, 2012;37(4):401-9.

4. European Monitoring Centre for Drug and Drug Addiction. Best practice portal [internet]. [citado 2016 jul 5]. Disponible en: http://www.emcdda.europa.eu/best-practice

5. Rodríguez LE. Guía de buenas prácticas en los programas de incorporación sociolaboral de las entidades de la unAD. Madrid: unAD; 2005.

6. Nieves Y. Buenas prácticas en el uso de redes sociales en los programas de prevención con población juvenil. Madrid: Fundación Atenea; 2015.

7. Federación española de municipios y provincias. Primer catálogo de buenas prácticas en drogodependencias. España: Federación española de municipios y provincias; 2003.

8. Red Iberoamericana de ong que trabajan en drogodependencias (RIOD). Identificación y difusión de buenas prácticas de intervención de las ONG. Madrid: RIOD; 2016.

9. Gásquez M, García del Castillo J, Espada J. Eficacia de los programas de prevención escolar en función del agente preventivo: Profesores vs. Expertos. Salud Drogas, 2010;10(2):111-31.

10. García del Castillo J. Comparación de tres programas de prevención escolar para reducir el consumo de cannabis en función del tipo de aplicador. Universitas Psychologica, 2016;15(4):1-14. DoI: 10.11144/Javeriana.upsy15-4.cppe

11. Hernández EM. Evaluación de un programa de prevención del consumo de sustancias psicoactivas para la infancia. Salud Drogas, 2015;15(1):67-78.

12. Foxcroft DR, Tsertsvadze A. Universal multi-component prevention programs for alcohol misuse in young people. Cochrane Database Syst Rev. 2011;7(9). Dor: 10.1002/14651858.CD009307

13. Jones L, Sumnall H, Witty K, Wareing M, McVeigh J, Bellis M. A review of community-based interventions to reduce substance misuse among vulnerable and disadvantaged young people. Liverpool: John Moores University; 2006. 
14. Fernández S, Nebot M, Jané M. Evaluación de la Efectividad de los Programas Escolares de Prevención del Consumo de Tabaco, Alcohol y Cannabis: ¿Qué nos dicen los MetaAnálisis? Rev Esp Salud Pública. 2002;3:175-87.

15. Gázquez M, García del Castillo JP, Espada JP. Características de los programas eficaces para la prevención escolar del consumo de drogas. Salud Drogas. 2009;9(2):185-208.

16. Mejía J, Pérez-Gomez A, Reyes-Rodríguez MF. Implementación y adaptación en Colombia del sistema preventivo Communities That Care. Adicciones. 2015;27(4):253-64.

17. Consorcio Internacional Sobre Políticas de Drogas IDPc. Guía Sobre Políticas de Drogas [internet]. 2012 [citado 2017 may 2]. Disponible en: http://fileserver.idpc.net/library/ Guia-sobre-politicas-de-drogas_Segunda-edicion.pdf

18. NIDA. Prevention Drug Use among Children and Adolescentes [internet]. 2003 [citado 2017 ene 30]. Disponible en: https://www.drugabuse.gov/sites/default/files/preventingdruguse_2.pdf

19. Burkhart G. ¿Confiamos demasiado en el valor de la cognición y de la Educación en la Prevención? Revista Española de Drogodependencias, 2015;40(4):61-70.

20. Milanese E. Tratamiento Comunitario. Manual de Trabajo I, 3. ${ }^{\mathrm{a}}$ ed. São Paulo: Associacao de Formcao e Reducacao Lua Nova; 2016.

21. Machin J, Merlo R. Milanese E. Redes Sociales y Farmacodependencias. Aportes para la intervención. México: ConAdic; 2010.

22. Cuijpers P. Peer-led and adult-led school drug prevention: A meta-analytic comparision. J Drug Educ, 2002;32(2):107-19.

23. Botvin GJ, Griffin KW. Long-term outcomes from Blueprints model programs: Life Skills Training. Presentado en: Blueprints for Violence Prevention conference. 2012 abr 1114; San Antonio, TX.

24. Becoña E. Bases Científicas de la prevención de las drogodependencias. Madrid: Delegación del Gobierno para el Plan Nacional sobre Drogas; 2002.

25. Gorman D. Does the Life Skills Training program reduce use of marijuana? Addict Res Theory, 2011;19(5):470-81. DoI: 10.3109/16066359.2011.557164

26. Kumpfer K, Alvarado R. Family-strengthening approaches for the prevention of youth problem behaviors. The American psychologist, 2003;58:457-65.

27. Hawkins JD, Catalano RF, Miller JY. Risk and protective factors for alcohol and other drug problems in adolescence and early adulthood: Implications for substance abuse prevention. Psychol Bull, 1992;112(1):64-105.

28. Programa de Cooperación entre América Latina y la Unión Europea en Políticas sobre Drogas (COPOLAD). Calidad y evidencia en reducción de la demanda de drogas, Madrid: COPOLAD; 2014.

29. Gásquez M, García del Castillo JA, Ruiz I. Importancia de la fidelidad en la implementación de programas escolares para prevenir el consumo de drogas. Salud Drogas, 2011;11(1):51-69.

30. Gómez JA, Luengo A, Romero E. Prevención del consumo de drogas en la escuela: cuatro años de seguimiento de un programa. Psicothema, 2002;14(4):685-92. 
31. Fraguela JA, Martin AL, Trinanes EA. Drug-Abuse prevention in the school: Four-year follow-up of a programme. Psychology in Spain, 2003;7:29-38.

32. Botvin GJ, Griffin KW, Diaz T, Scheier LM, Williams C, Epstein JA. Preventing illicit drug use in adolescents: Long-term follow-up data from a randomized control trial of a school population. Addict Behav, 2000;25:769-74.

33. Griffin KW, Botvin GJ, Nichols TR, Doyle MM. Effectiveness of a universal drug abuse prevention approach for youth at high risk for substance use initiation. Prev Med, 2003;36:1-7.

34. Griffin KW, Botvin GJ, Nichols TR. Long-term follow-up effects of a school-based drug abuse prevention program on adolescent risky driving. Prevention Science, 2004;5:207-12.

35. Quintero B, Ortega JA, Fuentes JA. Evaluación del Programa de prevención de drogodependencias Entre Todos. Aula de Encuentro, 2017; 16(2):130-58.

36. De Vicenzi A, Bareilles G. Promoción de la salud y prevención escolar del consumo de drogas en contextos de vulnerabilidad social. Educ. 2011;14(3):577-600.

37. Faggiano F, Minozzi S, Versino E, Buscemi D. Universal school-based prevention for illicit drug use. Cochrane Database of Systematic Reviews 2014;12. DOI: 10.1002/14651858. CD003020.pub3.

38. Comas D. ¿Qué es la evidencia científica y cómo utilizarla? Una propuesta para profesionales de la intervención. Madrid: Fundación Atenea; 2014. 